\title{
ОБ УСЛОВИЯХ АБСОЛЮТНОЙ ЧЕЗАРОВСКОЙ СУММИРУЕМОСТИ КРАТНЫХ ТРИГОНОМЕТРИЧЕСКИХ РЯДОВ ФУРЬЕ
}

\section{С. Битимхан}

\begin{abstract}
Получено необходимое и достаточное условие абсолютной $|C ; \bar{\beta}|_{\lambda}$-суммируемости почти всюду на $\mathbb{T}^{s}$ кратных тригонометрических рядов Фурье функций $f \in L_{\bar{q}}\left(\mathbb{T}^{s}\right)$, принадлежащих к обобщенным классам Бесова $B_{\bar{q}, s, \theta}^{\omega_{r}}$, где $\mathbb{T}^{s}=[0,2 \pi)^{s}, \bar{\beta}=\left(\beta_{1}, \beta_{2}, \ldots, \beta_{s}\right), \bar{q}=\left(q_{1}, q_{2}, \ldots, q_{s}\right), 1<q_{j} \leq 2, j=\overline{1, s}, 1 \leq \lambda \leq q_{s} \leq$ $\ldots \leq q_{1}, \lambda<\theta<\infty, 0 \leq \beta_{j}<1 / q_{j}^{\prime}=1-1 / q_{j}, j=\overline{1, s}, r \in \mathbb{N}, r>\sum_{j=1}^{s}\left(1 / q_{j}-\beta_{j}\right), \omega_{r}-$ функция типа модуля гладкости порядка $r$.

Ключевые слова: кратные тригонометрические ряды Фурье, абсолютная суммируемость, модуль гладкости, обобщенный класс Бесова.
\end{abstract}

S. Bitimkhan. Conditions of absolute Cesaro summability of multiple trigonometric Fourier series.

A necessary and sufficient condition of absolute $|C ; \bar{\beta}|_{\lambda}$-summability almost everywhere on $\mathbb{T}^{s}$ is obtained for multiple trigonometric Fourier series of functions $f \in L_{\bar{q}}\left(\mathbb{T}^{s}\right)$ from generalized Besov classes $B_{\bar{q}, s, \theta}^{\omega_{r}}$, where $\mathbb{T}^{s}=[0,2 \pi)^{s}, \bar{\beta}=\left(\beta_{1}, \beta_{2}, \ldots, \beta_{s}\right), \bar{q}=\left(q_{1}, q_{2}, \ldots, q_{s}\right), 1<q_{j} \leq 2, j=\overline{1, s}, 1 \leq \lambda \leq q_{s} \leq \ldots \leq q_{1}, \lambda<\theta<\infty$, $0 \leq \beta_{j}<1 / q_{j}^{\prime}=1-1 / q_{j}, j=\overline{1, s}, r \in \mathbb{N}, r>\sum_{j=1}^{s}\left(1 / q_{j}-\beta_{j}\right)$, and $\omega_{r}$ is a function of the type of modulus of smoothness of order $r$.

Keywords: multiple trigonometric Fourier series, absolute summability, modulus of smoothness, generalized Besov class.

MSC: $42 \mathrm{~A} 24$

DOI: $10.21538 / 0134-4889-2019-25-2-42-47$

Пусть $\mathbb{R}^{s}-s$-мерное евклидово пространство точек $\bar{x}=\left(x_{1}, x_{2}, \ldots, x_{s}\right), s \in \mathbb{N}$;

$\mathbb{T}^{s}=\left\{\bar{x} \in \mathbb{R}^{s}: 0 \leq x_{j}<2 \pi, j=\overline{1, s}\right\} ; L_{\bar{q}}\left(\mathbb{T}^{s}\right)$ - пространство всех измеримых $2 \pi$ периодических по каждой переменной $x_{j}, j=\overline{1, s}$, функций $f(\bar{x})=f\left(x_{1}, x_{2}, \ldots, x_{s}\right)$, для которых

$$
\|f\|_{\bar{q}, s}=\left(\int_{0}^{2 \pi}\left[\cdots\left[\int_{0}^{2 \pi}|f(\bar{x})|^{q_{1}} d x_{1}\right]^{q_{2} / q_{1}} \cdots\right]^{q_{s} / q_{s-1}} d x_{s}\right)^{1 / q_{s}}<\infty,
$$

где $\bar{q}=\left(q_{1}, q_{2}, \ldots, q_{s}\right), 1 \leq q_{j}<\infty, j=\overline{1, s}$ (в случае $q_{1}=q_{2}=\ldots=q_{s}=q$ полагаем $\left.\|f\|_{\bar{q}, s} \equiv\|f\|_{q, s}\right)$

$E_{n, \infty}^{(j)}(f)_{\bar{q}, s}$ - частное наилучшее приближение функции $f(\bar{x}) \in L_{\bar{q}}\left(\mathbb{T}^{s}\right)$ тригонометрическими полиномами порядка не выше $n \in \mathbb{Z}_{+}$по переменной $x_{j}, j=\overline{1, s}$ (см., например, [1, гл. II, п. 2.2]);

$\omega_{r}^{(j)}(f ; \delta)_{\bar{q}, s}-$ частный модуль гладкости функции $f \in L_{\bar{q}}\left(\mathbb{T}^{s}\right)$ порядка $r \in \mathbb{N}$ по переменной $x_{j}, j=\overline{1, s}$ (см., например, [1, гл. III, пп. 3.4.34, 3.12.5]):

$$
\omega_{r}^{(j)}(f ; \delta)_{\bar{q}, s}=\sup \left\{\left\|\Delta_{h, x_{j}}^{r} f(\cdot)\right\|_{\bar{q}, s}: h \in \mathbb{R}^{1},|h| \leq \delta\right\}, \quad \delta \in[0,+\infty),
$$

где

$$
\Delta_{h, x_{j}}^{r} f(\bar{x})=\sum_{\nu=0}^{r}(-1)^{r-\nu}\left(\begin{array}{l}
r \\
\nu
\end{array}\right) f\left(x_{1}, \ldots, x_{j-1}, x_{j}+h, x_{j+1}, \ldots, x_{s}\right), \quad\left(\begin{array}{l}
r \\
\nu
\end{array}\right)=\frac{r !}{\nu !(r-\nu) !}, \quad \nu=\overline{0, r}
$$


Положим $\gamma_{i}(n x)=\{\cos n x, i=1 ; \sin n x, i=2\}$, где $x \in \mathbb{R}^{1}, n \in \mathbb{N}$. Рассмотрим кратный тригонометрический ряд

$$
\sum_{\bar{n} \geq \overline{1}} B_{\bar{n}}(\bar{x})=\sum_{n_{1}=1}^{\infty} \ldots \sum_{n_{s}=1}^{\infty} B_{n_{1}, \ldots, n_{s}}\left(x_{1}, \ldots, x_{s}\right),
$$

где

$$
B_{\bar{n}}(\bar{x})=\sum_{\overline{1} \leq \bar{i} \leq \overline{2}} a_{\bar{n}}^{(\bar{i})} \prod_{\nu=1}^{s} \gamma_{i_{\nu}}\left(n_{\nu} x_{\nu}\right), \quad a_{\bar{n}}^{(\bar{i})} \in \mathbb{R}^{1} \equiv \mathbb{R} .
$$

Для заданных $n \in \mathbb{N}$ и $\beta \in(-1,+\infty)$ положим $A_{n}^{(\beta)}=(\beta+1)(\beta+2) \ldots(\beta+n) / n$ !. Сумма

$$
\sigma_{\bar{n}}^{(\bar{\beta})}(\bar{x})=\sum_{\overline{1} \leq \bar{k} \leq \bar{n}} \prod_{j=1}^{s} A_{n_{j}-k_{j}}^{\left(\beta_{j}-1\right)}\left(A_{n_{j}}^{\left(\beta_{j}\right)}\right)^{-1} B_{\bar{k}}(\bar{x}), \quad \bar{n}=\left(n_{1}, \ldots, n_{s}\right),
$$

называется $(C ; \bar{\beta}) \equiv\left(C ; \beta_{1}, \ldots, \beta_{s}\right)$ средним (или средним по Чезаро) ряда (1).

Для чисел $b_{\bar{n}}$ определим смешанную разность $\Delta b_{\bar{n}}$ следующим образом:

$$
\Delta b_{\bar{n}}=\sum_{\eta_{1}=0}^{1} \ldots \sum_{\eta_{s}=0}^{1}(-1)^{s-|\bar{\eta}|} b_{\left(n_{1}-1+\eta_{1}, \ldots, n_{s}-1+\eta_{s}\right)}=\sum_{\overline{0} \leq \bar{\eta} \leq \overline{1}}(-1)^{s-|\bar{\eta}|} b_{\bar{n}-\overline{1}+\bar{\eta}}
$$

$\left(\bar{\eta}=\left(\eta_{1}, \ldots, \eta_{s}\right), \eta_{i} \in\{0,1\}, \quad i=\overline{1, s} ;|\bar{\eta}|=\eta_{1}+\ldots+\eta_{s}\right)$.

Для заданного числа $\lambda \geq 1$ ряд (1) называется $|C ; \bar{\beta}|_{\lambda}$-суммируемым (или абсолютно суммируемым по Чезаро) в точке $\bar{x} \in \mathbb{T}^{s}$, если

$$
\sum_{\bar{n} \geq \overline{1}}\left|\Delta \sigma_{\bar{n}}^{(\bar{\beta})}(\bar{x})\right|^{\lambda} \prod_{j=1}^{s} n_{j}^{\lambda-1}<\infty .
$$

(см., например, [2-4] - случай $\lambda=1 ;[5]-$ случай $s=1, \lambda \geq 1$ ).

Исследованию условий $|C ; \bar{\beta}|_{\lambda}$-суммируемости при $\lambda=1$ кратных тригонометрических рядов Фурье функций $f \in L_{2}\left(\mathbb{T}^{s}\right)$ в терминах поведения величин $E_{n, \infty}^{(j)}(f)_{2, s}$ и $\omega_{r}^{(j)}(f ; 1 / n)_{2, s}$ $(j=\overline{1, s}$ ) посвящены работы $[3 ; 4 ; 6]$ (см. в них также библиографию, где приведены ссылки на более ранние работы, связанные с рассматриваемой тематикой). В случае $s=1$ и $\lambda \geq 1$ наиболее разностороннее исследование вопроса $|C ; \alpha|_{\lambda}$-суммируемости $(\alpha>-1)$ рядов Фурье функций $f \in L_{q}(T)(1<q \leq 2)$ с детальным обзором ранее известных результатов было проведено И. Салаи в [5].

Для дальнейшего изложения нам понадобятся следующие обозначения:

$$
E_{\bar{q}, s}^{\varepsilon}=\left\{f \in L_{\bar{q}}\left(\mathbb{T}^{s}\right): E_{n, \infty}^{(j)}(f)_{\bar{q}, s}=O\left(\varepsilon_{n}\right), n \in \mathbb{N}, j=1,2, \ldots, s\right\},
$$

где $\varepsilon=\left\{\varepsilon_{n}\right\}_{n=1}^{\infty}$ - заданная последовательность положительных чисел, монотонно убывающая к 0 при $n \rightarrow \infty$;

$$
H_{\bar{q}, s}^{\omega_{r}}=\left\{f \in L_{\bar{q}}\left(T^{s}\right): \omega_{r}^{(j)}(f ; \delta)_{\bar{q}, s}=O\left(\omega_{r}(\delta)\right), \delta \in(0,1], j=1, \ldots, s\right\},
$$

где $\omega_{r}(\delta)$ - функция типа модуля гладкости порядка $r \in \mathbb{N}: \omega_{r}(\delta) \rightarrow 0=\omega_{r}(0)$ при $\delta \rightarrow 0$, $\omega_{r}(\delta)$ непрерывна и не убывает на отрезке $[0,1]$, для некоторого постоянного $C(r)>0$ и всех $0<\delta_{1}<\delta_{2} \leq 1$ выполняется неравенство $\delta_{2}^{-r} \omega_{r}\left(\delta_{2}\right) \leq C(r) \delta_{1}^{-r} \omega_{r}\left(\delta_{1}\right)$.

Кроме того, нам понадобится также определение обобщенного класса О.В. Бесова $B_{\bar{q}, s, \theta}^{\omega_{r}}$ (см., например, $\left[7\right.$, разд. $\left.\left.1^{\circ}\right]\right)$ :

$$
B_{\bar{q}, s, \theta}^{\omega_{r}}=\left\{f \in L_{\bar{q}}\left(\mathbb{T}^{s}\right):\left(\int_{0}^{1}\left(\frac{\omega_{r}^{(j)}(f ; t)_{\bar{q}, s}}{\omega_{r}(t)}\right)^{\theta} \frac{d t}{t}\right)^{1 / \theta}<\infty, j=1,2, \ldots, s\right\},
$$


где $1 \leq \theta \leq \infty$. В случае $\theta=\infty$ класс $B_{\bar{q}, s, \infty}^{\omega_{r}}=H_{\bar{q}, s}^{\omega_{r}}$. Если $q_{1}=q_{2}=\ldots=q_{s}=q$, то вместо $E_{\bar{q}, s}^{\varepsilon}$ и $H_{\bar{q}, s}^{\omega_{r}}$ будем писать соответственно $E_{q, s}^{\varepsilon}$ и $H_{q, s}^{\omega_{r}}$.

При наличии ограничений на параметры $1<q \leq 2,1 \leq \lambda \leq q, 0 \leq \beta_{j}<1 / q^{\prime}=1-1 / q$, $j=\overline{1, s}, r>s / q-\sum_{j=1}^{s} \beta_{j}$ в работе [8, теоремы 2 и 3] найдены необходимые и достаточные условия на последовательность $\varepsilon=\left\{\varepsilon_{n}\right\}_{n=1}^{\infty}$ (на функцию $\left.\omega_{r}(\delta)\right)$ для $|C ; \bar{\beta}|_{\lambda}$-суммируемости почти всюду на $\mathbb{T}^{s}$ ряда Фурье каждой функции $f \in E_{q, s}^{\varepsilon}$ (соответственно $f \in H_{q, s}^{\omega_{r}}$ ).

Утверждения теорем 2 и 3 из [8] позднее были обобщены на случай классов функций со смешанной нормой $E_{\bar{q}, s}^{\varepsilon}$ и $H_{\bar{q}, s}^{\omega_{r}}$ в работе [9, теорема 3 и теорема 4]. В дальнейшем $C_{j}(r, s, \ldots)$, где $j \in \mathbb{N}$, обозначают положительные числа, значения которых зависят только от указанных в скобках параметров; в случае, когда понятно, от каких параметров зависят упомянутые числа, используется обозначение $C_{j}$.

Основным результатом настоящей работы является следующее утверждение.

Теорема. Пусть $1<q_{j} \leq 2, j=\overline{1, s}, 1 \leq \lambda \leq q_{s} \leq \ldots \leq q_{1}, \lambda<\theta<\infty, 0 \leq \beta_{j}<1 / q_{j}^{\prime}=$ $1-1 / q_{j}, j=\overline{1, s}, r>\Re \equiv \sum_{j=1}^{s}\left(1 / q_{j}-\beta_{j}\right)$. Тогда для $|C ; \bar{\beta}|_{\lambda}$-суммируемости почти всюду на $\mathbb{T}^{s}$ ряда Фурье каждой функции $f \in B_{\bar{q}, s, \theta}^{\omega_{r}}$ необходимо и достаточно выполнения условия

$$
\int_{0}^{1} \omega_{r}^{\frac{\lambda \theta}{\theta-\lambda}}(t) \cdot t^{-\left(\frac{\lambda \theta}{\theta-\lambda} \circledast+1\right)} d t<\infty .
$$

Д о к а з а т е л ь с т в о. Достаточность. Пусть $f \in B_{\bar{q}, s, \theta}^{\omega_{r}}$ и выполняется (2). В силу неравенства (см., например, [1, гл. V, п. 5.3.1, неравенство (1)])

$$
E_{n, \infty}^{(j)}(f)_{\bar{q}, s} \leq C_{1}(r, s) \omega_{r}^{(j)}(f ; 1 / n)_{\bar{q}, s}, \quad f \in L_{\bar{q}}\left(\mathbb{T}^{s}\right), \quad 1 \leq q_{j}<\infty, \quad j=\overline{1, s},
$$

имеем

$$
\begin{gathered}
\sum_{n=1}^{\infty} n^{\lambda æ-1}\left(E_{n, \infty}^{(j)}(f)_{\bar{q}, s}\right)^{\lambda} \asymp \sum_{k=0}^{\infty} 2^{k \lambda æ}\left(E_{2^{k}, \infty}^{(j)}(f)_{\bar{q}, s}\right)^{\lambda} \\
\leq C_{1}^{\lambda}(r, s) \sum_{k=0}^{\infty} 2^{k \lambda æ}\left(\omega_{r}^{(j)}\left(f ; 2^{-k}\right)_{\bar{q}, s}\right)^{\lambda}=C_{1}^{\lambda}(r, s) \sum_{k=0}^{\infty} 2^{k \lambda æ} \omega_{r}^{\lambda}\left(2^{-k}\right)\left(\frac{\omega_{r}^{(j)}\left(f ; 2^{-k}\right)_{\bar{q}, s}}{\omega_{r}\left(2^{-k}\right)}\right)^{\lambda} .
\end{gathered}
$$

Поскольку $\lambda<\theta$, то, применяя неравенство Гельдера с показателями $\tau=\theta / \lambda>1, \tau^{\prime}=\theta /(\theta-\lambda)$ $\left(1 / \tau+1 / \tau^{\prime}=1\right)$, получим

$$
\begin{aligned}
& \sum_{n=1}^{\infty} n^{\lambda æ-1}\left(E_{n, \infty}^{(j)}(f)_{\bar{q}, s}\right)^{\lambda}
\end{aligned}
$$

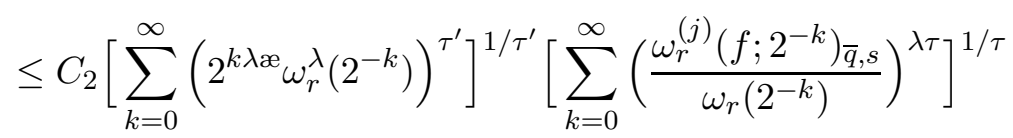

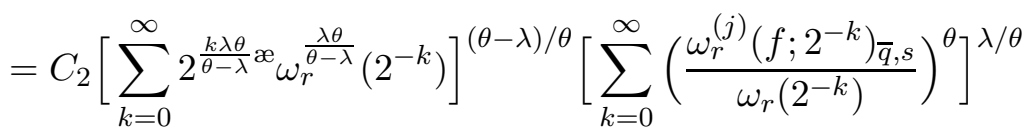

$$
\begin{aligned}
& \leq C_{3}\left[\int_{0}^{1} \omega_{r}^{\frac{\lambda \theta}{\theta-\lambda}}(t) \cdot t^{-\left(\frac{\lambda \theta}{\theta-\lambda} \circledast+1\right)} d t\right]^{(\theta-\lambda) / \theta}\left[\int_{0}^{1}\left(\frac{\omega_{r}^{(j)}(f ; t) \bar{q}, s}{\omega_{r}(t)}\right)^{\theta} \frac{d t}{t}\right]^{\lambda / \theta} .
\end{aligned}
$$

Из полученной оценки следует, что

$$
\sum_{n=1}^{\infty} n^{\lambda æ-1}\left(E_{n, \infty}^{(j)}(f)_{\bar{q}, s}\right)^{\lambda}<\infty, \quad j=\overline{1, s}
$$


откуда в силу теоремы 2 из [9] ряд Фурье функции $f \in B_{\bar{q}, s, \theta}^{\omega_{r}}$ является $|C ; \bar{\beta}|_{\lambda \text {-суммируемым }}$ почти всюду на $\mathbb{T}^{s}$.

Необходимостъ. Допустим, что ряд Фурье каждой функции $f \in B_{\bar{q}, s, \theta}^{\omega_{r}}$ является $|C ; \bar{\beta}|_{\lambda^{-}}$ суммируемым почти всюду на $\mathbb{T}^{s}$, однако условие $(2)$ не выполняется. Покажем, что в этом случае найдется функция $g_{0}(\bar{x}) \in B_{\bar{q}, s, \theta}^{\omega_{r}}$, ряд Фурье которой не будет $|C ; \bar{\beta}|_{\lambda}$-суммируемым почти всюду на $\mathbb{T}^{s}$.

Построение функции $g_{0}(\bar{x})$ проведем, следуя схеме, приведенной в статье $\left[7\right.$, разд. $3^{\circ}$, доказательство необходимости теоремы, п. 1]. Вначале отметим, что расходимость интеграла (2) равносильна расходимости ряда

$$
\sum_{k=0}^{\infty} 2^{\frac{k \lambda \theta}{\theta-\lambda} \omega_{r}^{\frac{\lambda \theta}{\theta-\lambda}}}\left(2^{-k}\right)=+\infty
$$

В обозначениях $d_{k}=2^{k æ} \omega_{r}\left(2^{-k}\right), p=\lambda \theta /(\theta-\lambda)$ условие (3) примет вид $\sum_{k=0}^{\infty} d_{k}^{p}=+\infty$. Тогда найдется числовая последовательность $\left\{\varepsilon_{k}\right\}_{k=0}^{\infty}\left(0<\varepsilon_{k} \downarrow 0\right.$ при $\left.k \rightarrow \infty\right)$, удовлетворяющая условиям (см. $\left[7\right.$, раз. $3^{\circ}$, п. 1 , свойства $\left.\left.(22)\right]\right)$ :

$$
\text { 1) } \left.\varepsilon_{k} d_{k}^{p / \lambda} \leq 1, \quad \text { 2) } \sum_{k=0}^{\infty} d_{k}^{p} \varepsilon_{k}^{\theta}<\infty, \quad 3\right) \sum_{k=0}^{\infty} d_{k}^{p} \varepsilon_{k}^{\lambda}=\infty .
$$

Положим (равенство понимается в смысле сходимости в метрике $L_{\bar{q}}\left(\mathbb{T}^{s}\right)$ )

$$
g_{0}(\bar{x})=\sum_{k=0}^{\infty} d_{k}^{p / \lambda} \varepsilon_{k} 2^{-k \mu} \prod_{j=1}^{s} \sum_{n_{j}=2^{k}+1}^{2^{k+1}} \cos n_{j} x_{j}, \quad \mu=\sum_{j=1}^{s}\left(1-\beta_{j}\right) .
$$

В силу известной оценки нормы одномерного ядра Дирихле (см., например, $[10, \S 4$, доказательство теоремы 5 в части "необходимость", неравенство (4.17) и неравенство после формулы (4.22)]) имеем

$$
\left\|\prod_{j=1}^{s} \sum_{n_{j}=2^{k}+1}^{2^{k+1}} \cos n_{j} x_{j}\right\|_{\bar{q}, s}=\prod_{j=1}^{s}\left\|\sum_{n_{j}=2^{k}+1}^{2^{k+1}} \cos n_{j} x_{j}\right\|_{q_{j}, 1} \leqslant C_{4}(\bar{q}, s) 2^{k \sum_{j=1}^{s}\left(1-1 / q_{j}\right)}, \quad k \in \mathbb{Z}_{+},
$$

откуда, учитывая также условие 1 ) в (4) и условие $\beta_{j}<1 / q_{j}^{\prime}, j=\overline{1, s}$, получим

$$
\begin{gathered}
\left\|g_{0}\right\|_{\bar{q}, s} \leqslant \sum_{k=0}^{\infty} d_{k}^{p / \lambda} \varepsilon_{k} 2^{-k \mu}\left\|\prod_{j=1}^{s} \sum_{n_{j}=2^{k}+1}^{2^{k+1}} \cos n_{j} x_{j}\right\|_{\bar{q}, s} \leq C_{4} \sum_{k=0}^{\infty} d_{k}^{p / \lambda} \varepsilon_{k} 2^{-k \sum_{j=1}^{s}\left(1-\beta_{j}\right)} 2^{k \sum_{j=1}^{s}\left(1-1 / q_{j}\right)} \\
=C_{4} \sum_{k=0}^{\infty} d_{k}^{p / \lambda} \varepsilon_{k} 2^{-k æ} \leq C_{4} \sum_{k=0}^{\infty} 2^{-k æ}<\infty .
\end{gathered}
$$

Из данной оценки следует, что ряд (5) действительно сходится в метрике $L_{\bar{q}}\left(\mathbb{T}^{s}\right)$ к некоторой функции $g_{0} \in L_{\bar{q}}\left(\mathbb{T}^{s}\right)$.

Покажем теперь, что $g_{0} \in B_{\bar{q}, s, \theta}^{\omega_{r}}$. Положим

$$
\delta_{k}\left(g_{0} ; \bar{x}\right)=\sum_{2^{k}+1 \leq \bar{n} \leq 2^{k+1}} B_{\bar{n}}\left(g_{0}, \bar{x}\right) .
$$

Воспользуемся утверждением (см. в $\left[7\right.$, разд. $2^{\circ}$, п. 1, с. 328]), согласно которому $g_{0} \in B_{\bar{q}, s, \theta}^{\omega_{r}}$ тогда и только тогда, когда $\sum_{k=0}^{\infty}\left(\omega_{r}^{-1}\left(2^{-k}\right)\left\|\delta_{k}\left(g_{0} ; \cdot\right)\right\|_{\bar{q}, s}\right)^{\theta}<\infty$. Учитывая оценку (6), определение $d_{k}$ и условие 2$)$ в (4), имеем

$$
\sum_{k=0}^{\infty}\left(\omega_{r}^{-1}\left(2^{-k}\right)\left\|\delta_{k}\left(g_{0} ; \cdot\right)\right\|_{\bar{q}, s}\right)^{\theta}=\sum_{k=0}^{\infty}\left(\omega_{r}^{-1}\left(2^{-k}\right) d_{k}^{p / \lambda} \varepsilon_{k} 2^{-k \mu}\right)^{\theta}\left\|\prod_{j=1}^{s} \sum_{n_{j}=2^{k}+1}^{2^{k+1}} \cos n_{j} x_{j}\right\|_{\bar{q}, s}^{\theta}
$$




$$
\begin{gathered}
\leq C_{4}^{\theta} \sum_{k=0}^{\infty}\left(\omega_{r}^{-1}\left(2^{-k}\right) d_{k}^{p / \lambda} \varepsilon_{k} 2^{-k æ}\right)^{\theta}=C_{4}^{\theta} \sum_{k=0}^{\infty}\left(d_{k}^{p / \lambda-1} \varepsilon_{k}\right)^{\theta} \\
=C_{4}^{\theta} \sum_{k=0}^{\infty} d_{k}^{\theta \lambda /(\theta-\lambda)} \varepsilon_{k}^{\theta}=C_{4}^{\theta} \sum_{k=0}^{\infty} d_{k}^{p} \varepsilon_{k}^{\theta}<\infty
\end{gathered}
$$

откуда следует, что $g_{0} \in B_{\bar{q}, s, \theta}^{\omega_{r}}$.

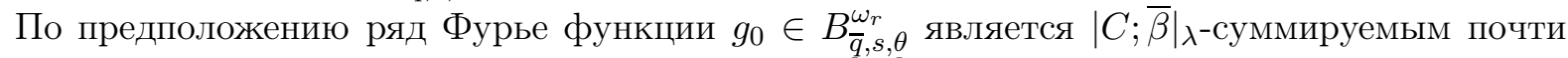
всюду на $\mathbb{T}^{s}$. Следовательно, согласно теореме 2 из [11] ряд

$$
\sum_{\bar{n} \geq \overline{1}}\left|a_{\bar{n}}\left(g_{0}\right)\right|^{\lambda} \prod_{j=1}^{s} n_{j}^{\lambda\left(1-\beta_{j}\right)-1}<\infty .
$$

Отметим, что утверждение теоремы 2 из [11] является распространением на многомерный случай для косинус-рядов соответствующего одномерного результата И. Салаи [5, разд. 1 , п. (i) теоремы 1].

Далее, согласно определению функции $g_{0}$, а также учитывая условие 3 ) в (4), получим

$$
\begin{gathered}
\sum_{\bar{n} \geq \overline{1}}\left|a_{\bar{n}}\left(g_{0}\right)\right|^{\lambda} \prod_{j=1}^{s} n_{j}^{\lambda\left(1-\beta_{j}\right)-1}=\sum_{k=0}^{\infty} \prod_{j=1}^{s} \sum_{n_{j}=2^{k}+1}^{2^{k+1}}\left|a_{\bar{n}}\left(g_{0}\right)\right|^{\lambda} \prod_{j=1}^{s} n_{j}^{\lambda\left(1-\beta_{j}\right)-1} \\
=\sum_{k=0}^{\infty}\left(d_{k}^{p / \lambda} \varepsilon_{k} 2^{-k \mu}\right)^{\lambda} \prod_{j=1}^{s} \sum_{n_{j}=2^{k}+1}^{2^{k+1}} n_{j}^{\lambda\left(1-\beta_{j}\right)-1}=\sum_{k=0}^{\infty} d_{k}^{p} \varepsilon_{k}^{\lambda} 2^{-k \lambda \mu} \prod_{j=1}^{s} \sum_{n_{j}=2^{k}+1}^{2^{k+1}} n_{j}^{\lambda\left(1-\beta_{j}\right)-1} \\
\geq C_{5} \sum_{k=0}^{\infty} d_{k}^{p} \varepsilon_{k}^{\lambda} 2^{-k \lambda \mu} \prod_{j=1}^{s} 2^{k\left(\lambda\left(1-\beta_{j}\right)-1\right)} 2^{k}=C_{5} \sum_{k=0}^{\infty} d_{k}^{p} \varepsilon_{k}^{\lambda} 2^{-k \lambda \mu} 2^{k \lambda \mu}=C_{5} \sum_{k=0}^{\infty} d_{k}^{p} \varepsilon_{k}^{\lambda}=+\infty,
\end{gathered}
$$

что противоречит сходимости ряда (7). Теорема доказана.

\section{СПИСОК ЛИТЕРАТУРЫ}

1. Тиман А.Ф. Теория приближения функций действительного переменного. Москва: Физматгиз, 1960. $624 \mathrm{c}$.

2. Тиман М.Ф. Об абсолютной сходимости и суммируемости рядов Фурье // Сообщения АН Грузинской ССР. 1961. Т. 26, № 6. С. 641-646.

3. Пономаренко Ю.А. Некоторые критерии абсолютной чезаровской суммируемости кратных рядов Фурье // Докл. АН СССР. 1963. Т. 152, № 6. С. 1305-1307.

4. Пономаренко Ю.А., Тиман М.Ф. Об абсолютной суммируемости кратных рядов Фурье // Укр. мат. журн. 1971. Т. 23, № 3. С. 346-361.

5. Салаи И. Об абсолютной суммируемости тригонометрических рядов // Мат. заметки. 1981. Т. 30, № 6. C. 823-837.

6. Тиман М.Ф., Пономаренко Ю.А. Некоторые критерии абсолютной суммируемости рядов Фурье // Исследования по современным проблемам конструктивной теории функций: сб. тр. Баку: Изд-во АН Азерб. ССР, 1965. С. 489-492.

7. Гольдман М.Л. О вложении обобщенных гельдеровых классов // Мат. заметки. 1972. Т. 12 , № 3. C. 325-336.

8. Акишев Г.А., Битимханулы С. Модули гладкости и абсолютная суммируемость кратных тригонометрических рядов // Мат. журн. Алматы. 2003. Т. 3, №1 (7). С. 5-14.

9. Bitimkhan S., Akishev G. The conditions of absolute summability of multiple trigonometric series // AIP Conf. Proc. 2015. Vol. 1676, no. 1, 020095. doi: 10.1063/1.4930521.

10. Ульянов П.Л. Об абсолютной и равномерной сходимости рядов Фурье // Мат. сб. 1967. Т. 72 (114), № 2. С. 193-225. 
11. Битимханулы С. Условие абсолютной суммируемости кратных тригонометрических рядов // Вестн. КазГУ. Сер.: математика, механика, информатика. 2001. № 1 (24). С. 3-11.

Поступила 31.08.2018

После доработки 27.03.2019

Битимхан Самат Принята к публикации 29.04.2019

канд. физ.-мат. наук,

Карагандинский государственный университет им. Е. А. Букетова,

г. Караганды, Казахстан

e-mail: bsamat10@mail.ru

\section{REFERENCES}

1. Timan A.F. Theory of approximation of functions of real variables. Macmillan, Pergamon Press, 1963. 631 p. Original Russian text published in Timan A.F. Teorija priblizhenija funkcij dejstvitel'nogo peremennogo. Moscow: Fizmatgiz Publ., 1960, 624 p.

2. Timan M.F. Absolute convergence and summability of Fourier series. Soobshch. Akad. Nauk Gruzin. SSR, 1961, vol. 26, no. 6, pp. 641-646 (in Russian).

3. Ponomarenko Yu.A. Criteria for absolute Cesaro summability of multiple Fourier series. Dokl. Akad. Nauk SSSR, 1963, vol. 152, no. 6, pp. 1305-1307 (in Russian).

4. Ponomarenko Yu.A., Timan M.F. On the absolute summability of Fourier multiple series. Ukr. Math. J., 1971, vol. 23, no. 3, pp. 291-304. doi: 10.1007/BF01085351.

5. Szalay I. Absolute summability of trigonometric series. Math. Notes, 1981, vol. 30, no. 6, pp. 912-919. doi: $10.1007 /$ BF01145770 .

6. Timan M.F., Ponomarenko Yu.A. Some criteria of absolute summability of Fourier series. In: Issled. Sovrem. Probl. Konstr. Teor. Funkts., sbornik trudov, Baku: AN Azerb. SSR, 1965, pp. 489-492 (in Russian).

7. Gol'dman M.L. On the inclusion of generalized Hölder classes. Math. Notes, 1972, vol. 12, no. 3, pp. 626-631. doi: 10.1007/BF01093999.

8. Akishev G.A., Bitimkhanuly S. Moduli of smoothness and the absolute summability of multiple trigonometric series. Mat. Zh. Almaty, 2003, vol. 3, no. 1, pp. 5-14 (in Russian).

9. Bitimkhan S., Akishev G. The conditions of absolute summability of multiple trigonometric series. AIP Conf. Proc., 2015, vol. 1676, no. 1, 020095. doi: 10.1063/1.4930521.

10. Ul'yanov P.L. Absolute and uniform convergence of Fourier series. Math. USSR-Sb., 1967, vol. 1, no. 2, pp. 169-197. doi: 10.1070/SM1967v001n02ABEH001973.

11. Bitimkhanuly S. Condition for the absolute summability of multiple trigonometric series. Vestnik Kazakh. Univ. Ser. Mat., Mekh., Inf., 2001, no. 1 (24), pp. 3-11 (in Russian).

Received August 31, 2018

Revised March 27, 2019

Accepted April 29, 2019

Samat Bitimkhan. Cand. Phys.-Math. Sci., Karagandy State University named after E. A. Buketov, Karagandy city, 100028 Kazakhstan, e-mail: bsamat10@mail.ru .

Cite this article as: S. Bitimkhan. Conditions of absolute Cesaro summability of multiple trigonometric Fourier series, Trudy Instituta Matematiki i Mekhaniki URO RAN, 2019, vol. 25, no. 2, pp. 42-47. 